Revue des patrimoines

40 | 2019

Lits historiques. Première anthologie des lits européens du XVe au XIXe siècle

\title{
Le vocabulaire des lits de la Renaissance en France à la lumière des sources
}

Renaissance Beds in France, Questions of Vocabulary Raised by the Sources

\section{Muriel Barbier}

\section{(2) OpenEdition \\ Journals}

Édition électronique

URL : http://journals.openedition.org/insitu/23340

DOI : $10.4000 /$ insitu. 23340

ISSN : $1630-7305$

Éditeur

Ministère de la Culture

Référence électronique

Muriel Barbier, « Le vocabulaire des lits de la Renaissance en France à la lumière des sources », In Situ [En ligne], 40 | 2019, mis en ligne le 10 septembre 2019, consulté le 04 octobre 2019. URL : http:// journals.openedition.org/insitu/23340 ; DOI : 10.4000/insitu.23340

Ce document a été généré automatiquement le 4 octobre 2019.

\section{cc) $(9)$}

In Situ Revues des patrimoines est mis à disposition selon les termes de la licence Creative Commons Attribution - Pas d'Utilisation Commerciale - Pas de Modification 4.0 International. 


\title{
Le vocabulaire des lits de la
} Renaissance en France à la lumière des sources

Renaissance Beds in France, Questions of Vocabulary Raised by the Sources

\author{
Muriel Barbier
}

Dans son Blason du lit, Gilles Corrozet offre un portrait poétique du meuble qu'est le lit : Lict delicat, doulx et mollet, Lict de duvet si très douillet,

Lict de plume tant bonne et fine, Lict d'ung coustil blanc comme un cigne [...] Lict dont le chevet est si doulx Qu'il semble que ce soit veloux, Quand on y prent un bon repos... Lict dont les draps (comme on demande) Sentent la rose et la lavende [...] o lict le parement des chambres, Lict d'honneur plein de toute joye, Beau lict encourtiné de soye Pour musser la clarté qui nuict [...] Lict beneist de la main du prebstre [...] Lict d'amour sainct, lict honnorable [... $]^{1}$

1 (fig. 1) 
Figure 1

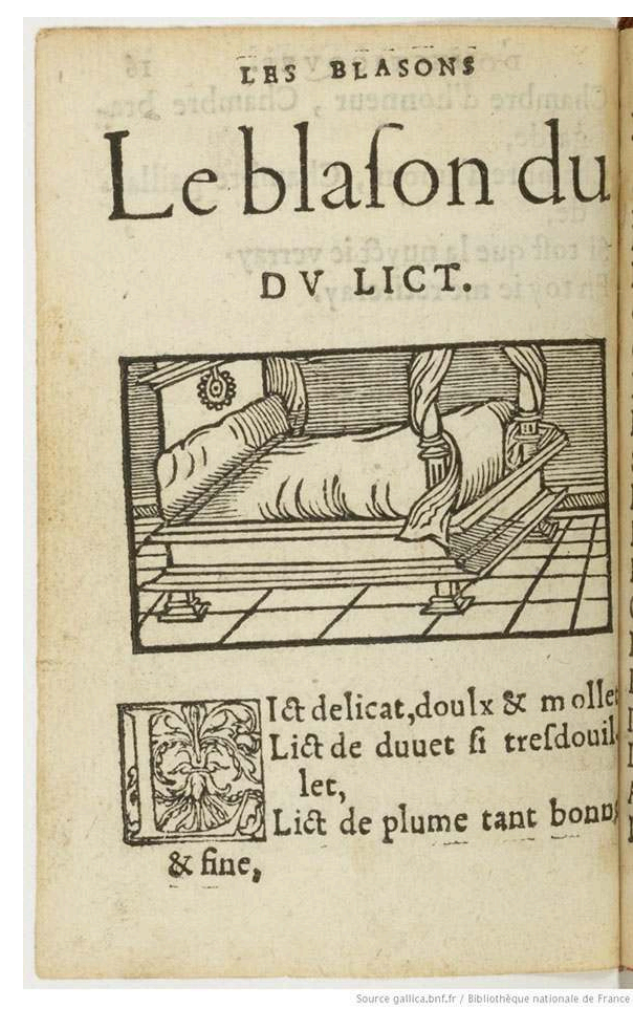

«Blason du lict », Les Blasons domestiques contenantz la décoration d'une maison honneste, et du mesnage estant en icelle,... Corrozet, Gilles, 1539. Paris, BnF, Réserve des livres rares, RES-YE-1380.

(C) BnF.

2 Au-delà de cette image idyllique, Gilles Corrozet révèle toute l'importance que revêtait le lit dans la France de la Renaissance en tant que théâtre de la naissance, de l'union matrimoniale et de la mort. Orthographié ici «lict», le mot désigne le meuble sur lequel on s'allonge, le meuble fait pour dormir mais dont la portée symbolique est nettement perceptible à travers les termes de « lict le parement », « lict d'honneur » et «lict honnorable ».

Ce meuble essentiel de la demeure est lui-même composé de différents éléments désignés par des termes spécifiques qui servent à le décrire, en particulier dans les inventaires après décès.

Or le $\mathrm{XvI}^{\mathrm{e}}$ siècle est en France une période transitoire durant laquelle le vocabulaire évolue en fonction des usages et des besoins; la terminologie est loin d'être systématique et l'orthographe n'est pas encore normée. À la lecture des sources, l'historien et le chercheur du xxI siècle se trouvent confrontés à un vocabulaire dont il est nécessaire de clarifier le sens.

\section{Ce que recouvre le vocable « lit»}

5 Plusieurs termes sont employés pour qualifier le lit. Pour le $\mathrm{Xv}^{\mathrm{e}}$ siècle, on constate déjà une diversité de désignations qui qualifient le même meuble, par exemple « lit » et « lit de camp $\rrbracket^{2}$. Ces deux termes semblent avoir été utilisés de façon indifférente, comme le révèlent les inventaires de la reine Anne de Bretagne (1495-1511) dans lesquels on peut 
lire: «Ladite Dame lez a prinse pour garnir les quenouilles du lit de camp [nous soulignons] de Monseigneur l'Archiduc $»^{3}$; or il paraît étonnant de trouver des quenouilles (colonnes) sur un lit de camp. À la lecture des sources, on comprend surtout que ces types de lit sont démontables. La vie encore itinérante de la société de Cour explique que l'on ait besoin de lits transportables d'une résidence à l'autre; le terme de « lit de camp » ne désigne donc pas nécessairement un lit sommaire de petite taille, tout comme le terme de "lit de parement» ne correspond pas à un lit fixe, malgré sa taille monumentale. Cette caractéristique du contexte curial apparaît nettement dans l'inventaire de 1542 (nouveau style) revu en 1551-1552 dans lequel le lit créé pour la venue de Charles Quint est mentionné ainsi : "ung lict portatif [nous soulignons] avec sa suyte qui fut faict de velours noir enrichy de fil d'or pour servir à l'empereur quant il passa par ce royaulme ${ }^{4}$.»

Quelle que soit la précision d'usage qui le suit (de camp, de parement, etc.), le vocable est - aux $\mathrm{xv}^{\mathrm{e}}$ et $\mathrm{xVI}^{\mathrm{e}}$ siècles - encore écrit indifféremment «lict» ou "lit»; c'est d'ailleurs la forme « lict» que retient Jean Nicot dans son Thresor de la langue françoyse en 1606 pour souligner l'étymologie latine du mot : « Lict, m. Vient du Latin Lectus, par apocope et mutation de e en i et signifie le receptacle où l'on se couche pour reposer de nuict ${ }^{5}$. » Cette première phrase de la définition semble bien refléter l'acception la plus commune du mot à la Renaissance : un meuble destiné au sommeil nocturne. Nicot poursuit sa définition ainsi : « Mais le mes-usage a approprié ce mot à ce que les Latins appellent Culcitra, et nous Coyte; Mais avec addition de ces mots, de plume, Un lict de plume. Et le reste, couche, ou chalict, ou bois de lict. » L'auteur souligne ici la confusion entre le meuble et certaines de ses parties, en l'occurrence la «couette »-qui au XVI siècle désigne un matelas de plumes - et la couche ou châlit qui correspond à la structure en bois du meuble.

7 En outre, le mot «lit» complété par un qualificatif textile désigne bien souvent uniquement la parure textile du lit et non tous ses éléments constitutifs (structure en bois, matelas et parure). Le lit, meuble d'apparat par excellence, est au $\mathrm{xvI}^{\mathrm{e}}$ siècle un véritable monument de textile, à tel point que les inventaires le mentionnent comme "lit de velours", "lit de damas", "lit brodé ", passant sous silence le bâti de menuiserie alors jugé secondaire et inventorié séparément des parties textiles. Plusieurs "chambres" sont mentionnées et décrites assez longuement dans l'inventaire de $1542^{6}$. Employé dans ce contexte, le terme de " chambre » ne désigne pas la chambre en tant que salle de la demeure mais un ensemble d'étoffes pouvant orner une pièce. Les « chambres textiles » de ces inventaires sont majoritairement destinées à des pièces de parement, "chambre vaste, solennelle, très richement décorée, où le Seigneur donne ses audiences, traite ses amis, reçoit les visites, les ambassades, etc. $»^{7}$

8 À la lecture des sources, la polysémie du terme « lit » peut donc prêter à confusion, car ce dernier désigne à la fois la structure en bois du meuble, mais le plus souvent l'ensemble des pièces textiles qui le recouvrent. Dans le premier cas, le mot "châlit ", orthographié de différentes manières, est préféré. Il en va de même pour le mot «quenouilles », terme qui désigne les supports en bois du ciel de lit situés à ses quatre angles et qui peuvent être décrites nues ou déjà gainées d'étoffe.

9 Ainsi, les sources d'archives, plus particulièrement les inventaires après décès, regorgent-elles de noms variés pour qualifier les parties textiles tandis que les châlits sont décrits moins précisément, révélant une réalité de la Renaissance : le bois de lit ne 
figure même pas pour un dixième de la valeur totale du lit, c'est le textile qui compte le plus.

\section{Des différentes parties du lit} plusieurs matelas superposés et d'une parure textile qui peut comprendre :

11 - un ciel ;

12 - des pentes, au nombre de trois ou sept;

13 - de trois ou six courtines, également appelées rideaux ;

14 - une pièce de dos qui, tombant le long du mur et isolant la couche, prend le nom de « chevet », « cheveciel », « dossier », « dosseret » ou « dociel »;

15 complétée par une « mante » ou « couverte »;

le $\mathrm{XVI}^{\mathrm{e}}$ siècle, les descriptions et mentions d'inventaire sont souvent partielles et n'incluent pas systématiquement les dimensions complètes. De plus, la terminologie employée pour les étoffes et les ornements brodés est également approximative. En outre, les inventaires ne sont pas encore systématiquement établis pièce par pièce ; au contraire, ils énumèrent les possessions du défunt par typologie ou en fonction des garde-meubles. Le lit ainsi répertorié ne l'est pas forcément dans la pièce à laquelle il était destiné. S'il n'est pas possible de se faire une idée précise des lits mentionnés dans les sources, ces dernières aident néanmoins à appréhender leur aspect général et à en préciser la terminologie.

\section{Le bâti}

La structure menuisée du lit apparaît dans les documents sous le vocable "châlit » orthographié "chaslit » ou "chaslict » c'est-à-dire "quasi chasse à lict. Car c'est comme l'enchassure du lict » selon Jean Nicot $^{8}$. Le terme châlit est relativement clair et désigne le bâti en bois du lit. Plus rarement cités que les éléments de parure textile, les châlits sont néanmoins présents dans les inventaires $\mathrm{du} \mathrm{xvI}{ }^{\mathrm{e}}$ siècle, notamment dans l'inventaire royal de 1542 avec par exemple « ung chaslict de bois doré bruny complet pour servir au ciel de satin cramoisy faict à L et aisles d'oiseau et de broderie de fil d'or "'. Les mots "couche » et "couchette » correspondent manifestement à des châlits de taille plus petite et à des lits courants. Pour Jean Nicot, la « couche » est le bois d'un lit $^{10}$. Cette différence de taille entre le lit et la couchette est confirmée par la plupart des sources. Par exemple, en 1579, Jean de Martineau, bourgeois parisien, se fait livrer un lit de 6 pieds de longs et 4 pieds et demi de large soit $194,88 \mathrm{~cm}$ et $146,16 \mathrm{~cm}$ et une couchette mesurant 5 pieds et demi de long sur 3 pieds de large ce qui correspond à $178,64 \mathrm{~cm}$ de long et $97,44 \mathrm{~cm}$ de large ${ }^{11}$. Durant tout le xvI ${ }^{\mathrm{e}}$ siècle, les lits mesurent un peu plus de 6 pieds $(194,88 \mathrm{~cm})$ et entre 4 et 5 pieds de large $(129,92 \mathrm{~cm}$ et $162,4 \mathrm{~cm})$. Ainsi, en 1575, un marchand parisien possédait-il deux lits : l'un de 6 pieds 4 pouces sur 
5 pieds 2 pouces $(205,68 \mathrm{~cm}$ sur $167,8 \mathrm{~cm})$ et l'autre de 6 pieds 2 pouces sur 4 pieds 2 pouces $(200,28 \mathrm{~cm}$ sur 133,32 cm). Autant de dimensions qui infirment le préjugé selon lequel les lits étaient petits et que l'on dormait assis; force est de constater que les tailles des lits du $\mathrm{XVI}^{\mathrm{e}}$ siècle étaient proches de celles d'aujourd'hui; les tailles standard étant $190 \mathrm{~cm}$ sur $120 \mathrm{~cm}$ et $200 \mathrm{~cm}$ sur $140 \mathrm{~cm}$ pour le lit double. La couchette correspondrait donc à notre lit une place de $190 \mathrm{~cm}$ sur $90 \mathrm{~cm}$.

En contexte royal ou princier, les lits peuvent atteindre des dimensions bien plus importantes en raison de l'importance symbolique du meuble dans le cérémonial de cour, en particulier lors des baptêmes princiers ou des pompes funèbres. Ainsi le lit de parement monté, en 1549, pour le baptême de Louis de France, duc d'Orléans, mesurait 10 pieds en carré $(325 \mathrm{~cm}$ sur $325 \mathrm{~cm})$ et 3 pieds et demi de haut $(114 \mathrm{~cm})^{12}$. Enfin, le lit créé pour le voyage de Charles Quint en France en 1539 mesurait $207 \mathrm{~cm}$ de long et $158 \mathrm{~cm}$ de large ${ }^{13}$.

Les différentes parties du châlit ne sont pas forcément décrites durant la première moitié du siècle car il s'agissait surtout de pièces de menuiserie simples destinées à être couvertes par le tissu. En revanche, dans la seconde moitié du Xvi ${ }^{\mathrm{e}}$ siècle, avec le développement de lits accordant une place plus importante à la sculpture, ces éléments sont parfois détaillés, ce qui permet de connaître le vocabulaire employé à cet effet. L'exemple d'un lit royal commandé à Noël Biard en 1584 est assez éloquent: le menuisier s'engage à faire "quatre tours de boys de lict qui seront de telle facon, grandeur, longueur et haulteur que sont ceulx qui sont de present en la chambre du Roy au chasteau du Louvre, fors et excepté que au lieu de colonnes y aura des balustres [...] Et pour ce faire, sera tenu led. Byat de fournir de boys et ferrure pour ce necessaire... ${ }^{14}$. La commande évoque des balustres à la place des colonnes : il s'agit des quatre montants servant à soutenir le ciel, plus souvent désignés sous le terme de "quenouilles»; ce dernier mot semble d'ailleurs réservé aux colonnes gainées de textile.

Recouvertes d'étoffe, les quenouilles sont rarement décrites isolément mais plus généralement mentionnées par groupes? paquets de quatre, à l'image de ces « quatre quenouilles et troys atybois, couvertz de satin cramoisy à cordelières de drap d'or frizé rapportez par-dessus $»^{15}$.

Dans certains cas, les bâtis de lit ont été cassés, et sont alors mentionnés en fin d'inventaire, ce qui donne quelques indications sur les bois employés comme ce «boys de lict de Caen de noyer [lire: lit de camp] sans aucun parement pour ce que les quenouilles se doivent vestir des foureaulx ci-devant enregistrez, cassé et rompu ${ }^{16}$.

Bien que la signification du terme châlit semble évidente, nous trouvons parfois des précisions pouvant paraître redondantes, comme dans l'inventaire de la duchesse de Valentinois Charlotte d'Albret, où il est question d'" ung chaslit de lict, ung buffet et ung petit banc $»^{17}$.

26 Les autres termes employés pour décrire les parties du châlit sont «tour de lit » et «bordure » pour les traverses.

27 Les menuisiers, fabricants des châlits, étaient tenus de fournir toutes les pièces nécessaires au maintien de la literie, en particulier les vis du châssis - qu'on appellerait aujourd'hui sommier - ainsi que les sangles garnissant ce châssis destinées à soutenir les matelas. Ces indications apparaissent dans un marché passé entre Pierre Langevin, compagnon menuisier, et Jean Malingre, avocat au Parlement de Paris, pour la 
confection de « deux lits de camp, c'est assavoir une couche et une couchette de boys de cormyer qui luy sera livré par led. Malingre, et ce de la façon et sorte qu'est faicte la couche de $\mathrm{M}^{\mathrm{e}}$ Francoys Frenicle, notaire, et faire oultre une patte ou bas desd. couches et au chevet une bordure daventage à chacune desd. couches, et de la longueur et largeur de celles dud. Malingre. Et sera tenu led. Langevin fornir à ses despens les enfonssures, sangles, ferrures, les chassis à vis de dessus de chesne desd. couches, et aussy des sangles à l'entour desd. couches couvertes de cuyr ${ }^{18}$.

\section{La literie}

Sur le châssis sanglé, les matelas sont superposés au nombre de trois ou plus (fig. 2).

Figure 2

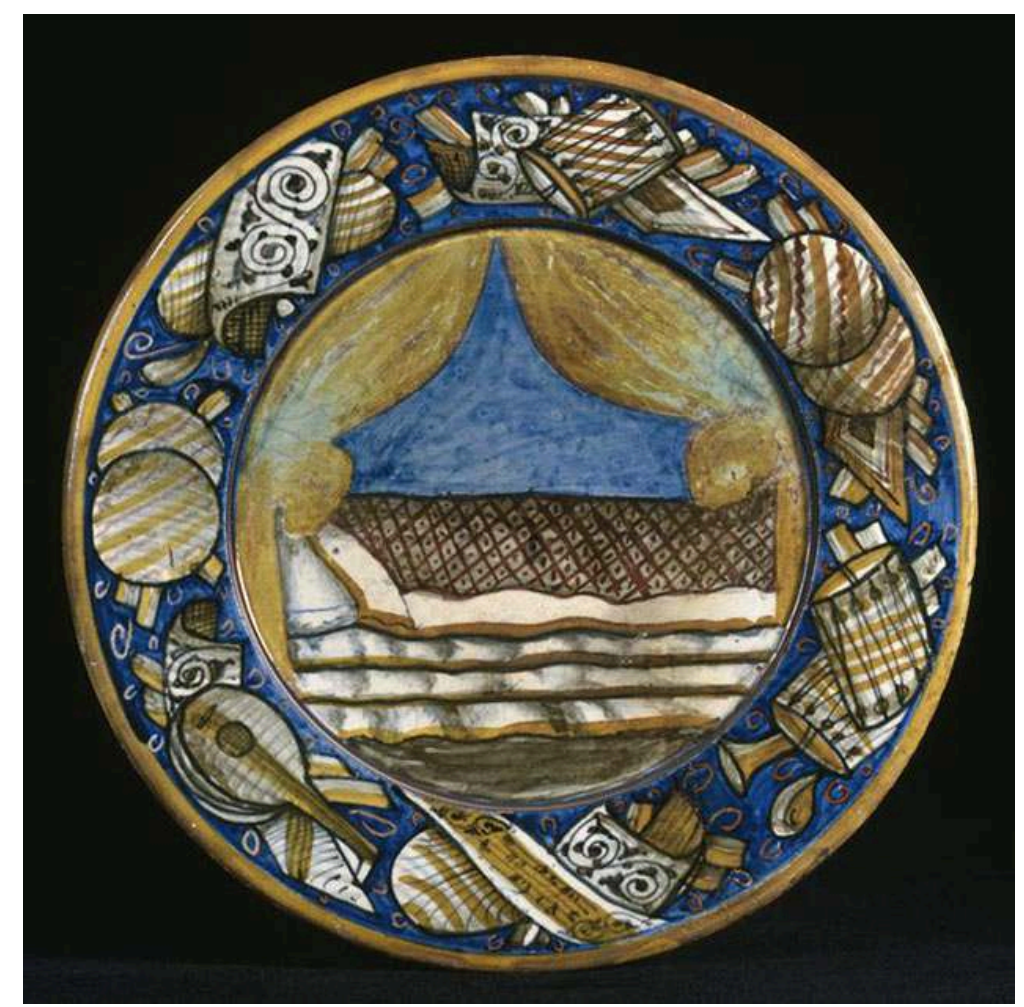

Castel Durante. Assiette : au centre, lit à trois épaisseurs de matelas. 1554, faïence, lustre métallique. Paris, musée du Louvre, département des Objets d'art, OA 1534.

Phot. Daniel Arnaudet. @ RMN-Grand Palais (musée du Louvre).

Dès le $\mathrm{XV}^{\mathrm{e}}$ siècle, les termes figurant dans les documents sont " pallasses ", " matraz » et «loder $»^{19}$. Selon le dictionnaire d'ancien français Huguet, la paillasse est faite de paille, le lodier est fait de deux étoffes cousues et garnies de laine et le matraz est destiné à recevoir le corps ${ }^{20}$. Au XvI ${ }^{\mathrm{e}}$ siècle, le mot «matelas » recouvre le même sens que de nos jours.

30 Les matelas sont cités à la fin des inventaires, avec les traversins et les carreaux; la nature de leur garniture est rarement donnée, en revanche le tissu qui les recouvre en précise l'aspect comme dans l'inventaire royal de 1542 où l'on trouve mention de plusieurs matelas en futaine - étoffe résistante dont la trame est en coton. Cette manière de faire est confirmée par le Premier dictionnaire de l'Académie française en ces 
termes : « On dit, Matelas de futaine, de satin, pour dire, Couvert de futaine, de satin ${ }^{21}$.» Dans ce même dictionnaire, le mot matelas est définit ainsi : "Espece de coüette remplie de laine, de cotton, de bourre, etc. ». Il semble donc que la garniture du matelas le distingue des autres couches de la literie que sont la couette et la paillasse.

À la fin de l'inventaire royal de 1542, sont justement mentionnées des " paillaces $»^{22}$. En 1694, le Dictionnaire de l'Académie française en donne pour définition : "Amas de paille enfermé dans de la toile pour servir de lit $\aleph^{23}$, acception déjà valable pour le $\mathrm{XvI}^{\mathrm{e}}$ siècle (fig. 3). La toile devait être de chanvre ou de lin.

Figure 3

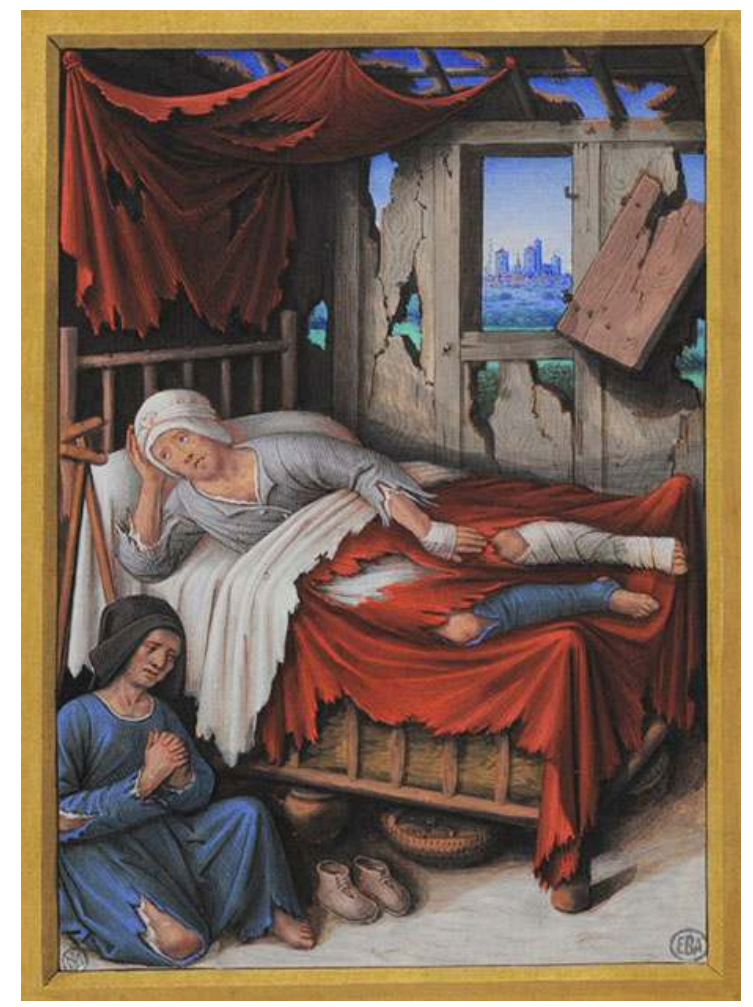

Jean Bourdichon. Les Quatre états de la société : L'homme misérable ou L'état de pauvreté, 1500-1510. Peinture sur parchemin. Paris, École nationale supérieure des beaux-arts, PC 47785, Mn.mas91 Creative commons (No copyright).

À ces paillasses et matelas de futaine viennent s'ajouter la "couette », "couete », «coette» ou «coitte». On en dénombre, par exemple, dix dans l'inventaire de Charlotte d'Albret. La couette est une sorte de sur-matelas garni de plumes d'oie ou de duvet. Elle rend le coucher plus confortable.

Traversins et carreaux - oreillers ou coussins - achèvent de rendre ces lits confortables. Comme les couettes, ils sont remplis de plume ou de duvet.

\section{Des parties textiles}

La parure d'un lit est évidemment fonction de la richesse de son propriétaire. Toutes les demeures ne sont donc pas meublées de lits à ciel. Néanmoins, nous prendrons ciaprès des exemples de lits royaux afin de pouvoir considérer tout le vocabulaire. Certains lits comprennent de nombreuses pièces textiles, dont l'inventaire royal de 
1542 donne des exemples très complets, à l'instar de la célèbre chambre dite « des Bucoliques ", d'après Virgile, et brodée sur les dessins de Matteo del Nassaro, provenant de l'ameublement de Louise de Savoie ${ }^{24}$. Outre les huit pièces murales en velours vert brodé, cette chambre comprend un grand lit dont chaque élément est détaillé :

- 11 Item ung grant ciel de parement, de pareil enrichissement et ouvraige, avec cinq histoires et taillettes, six penthes denviron demye aulne de large [...], enrichies de franges d'entretailleur faictes en façon de passure de haye de toille d'or et d'argent et de bouttons pendants de fil d'or et d'argent, doublée de toile noire de troys aulnes et demye de long et de sept lez de large, ouquel y a défault de plusieurs bouttons.

- 12 Ung dossier de pareil estoffe et ouvraige de deux aulnes troys quartz et demy de long et de cing lez de large, enrichy de cinq histoires et taillettes et de trois penthes doublées de demy lez de velours avec franges de pareille façon que le grand ciel dudict parement, ouquel a faulte de bouttons en plusieurs endroictz.

- 13 Item la queue du dict dosseret de troys aulnes deux tiers de hault et de cinq lez de large, enrichy de cinq histoires et taillettes avec un bort par le bas de doubles estocz comme celluy de la dicte tappicerie, doublé de satin de Bruge jaulne.

Plus tard dans le siècle, parmi d'autres lits, l'inventaire de Catherine de Médicis fait état d'« ung ciel à doubles pantes de velours blanc garny de son soubassement, trois grands rideaux de damas cafart avec une bonne grâce, quatre fourreaux de velours, une couverte de taffetas blanc piquée, une couverte de toille de Hollande piquée avec son traversin, [...] trois matelatz de fustaine blanche avec un boys de lit $»^{25}$.

Ces deux extraits d'inventaires royaux énumèrent pratiquement tous les éléments textiles pouvant orner un lit d'apparat en France au Xvi ${ }^{\mathrm{e}}$ siècle (fig. 4).

Figure 4

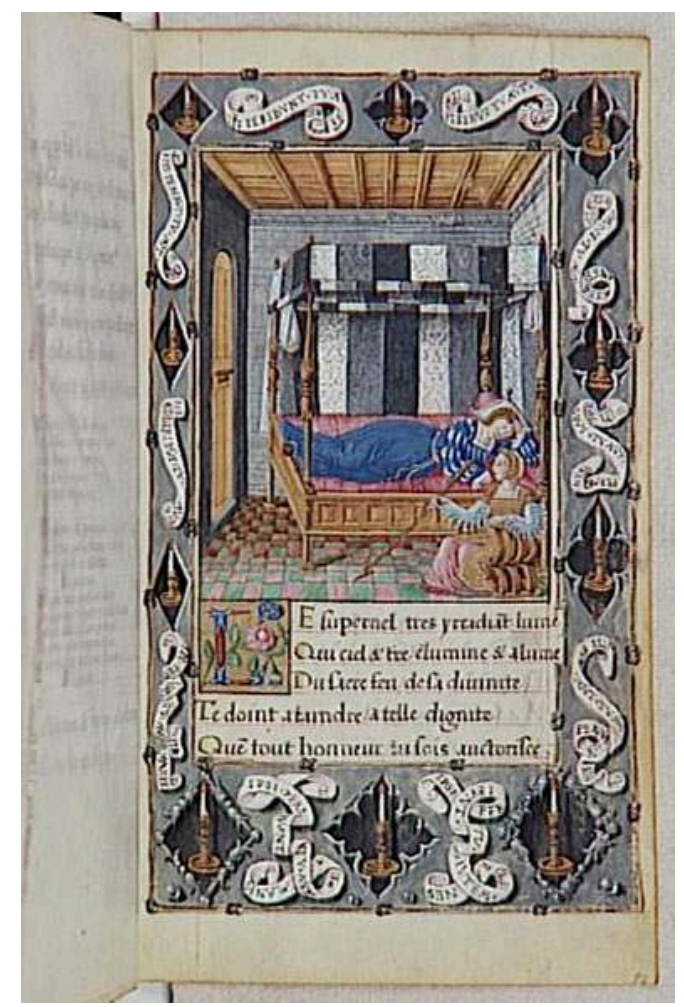

Anonyme français. L'Amant infortuné de François Habert : Fortune se rend auprès de la jeune dame, 1520-1530. Peinture sur parchemin. Chantilly, musée Condé, Ms 508, fol. 52.

Phot. René-Gabriel Ojéda. (c) RMN-Grand Palais (domaine de Chantilly). 


\section{Le ciel} apparaît comme un obstacle à la compréhension. En effet, le mot n'est pas systématiquement associé à un lit comme pour ce «ciel garny de pentes my party desd. couleur pour servir à tendre par-dessus l'oratoire » de Charles VIII au Plessis[-lèsTours $]^{26}$. Dans un oratoire, l'on comprend qu'il s'agit d'un ciel pour le dais de l'autel. Dans ce cas précis, la destination du ciel est indiquée et permet de de vérifier qu'il ne s'agit pas d'un ciel de lit. Dans d'autres cas, le positionnement du ciel dans l'inventaire aide à le rapprocher d'autres éléments comme le ciel en satin cramoisi brodé appelé «ciel de Milan », dans l'inventaire royal de 1542, dont la description est suivie de «troys panthes dudict ciel », du « dessus dudict ciel» puis de la « couverture dudit lict »; cette dernière mention invite à conclure que le ciel de Milan est un ciel de lit ${ }^{27}$. Cependant, les documents n'apportent pas toujours autant de précisions et mentionnent parfois indifféremment des ciels dont la destination demeure incertaine.

En 1694, le Premier dictionnaire de l'Académie française se fait l'écho de cette polysémie (1694) : «Ciel, signifie aussi, Le haut d'un lit » mais aussi « dais sous lequel on porte le Saint Sacrement $»^{28}$. Effectivement, dans les documents d'archives, «ciel» est fréquemment synonyme de dais, que ce soit dans un contexte sacré ou profane.

Dans le Thrésor de la langue françoyse, Jean Nicot indique simplement que «Ciel de lict » peut être rapproché de "Conopeum » qu'il traduit par moustiquaire, lit entouré d'une moustiquaire; ce qui n'est pas réellement la fonction du ciel de lit, superstructure de tissu à la dimension de la couche, tendue en hauteur et portée par les quenouilles.

En général, ces ciels sont décrits sans autres éléments de parure de lit mais ils peuvent être accompagnés de pentes et souvent d'un dosseret.

41 Une distinction doit être introduite entre le ciel et le pavillon, qui est suspendu au plafond de la pièce et non porté par les quenouilles. Au regard des documents écrits et iconographiques, le pavillon nous semble être conique et peut - dans certains exemples - doubler le ciel. Tel est le cas du lit créé pour la venue de Charles Quint en 1539, composé d'un châlit en noyer à quatre quenouilles gainées de velours noir brodé, un ciel à trois pentes doublées, le tour du lit, trois rideaux de damas noir et un pavillon du même damas noir qui venait couronner le ciel et marquer la présence impériale ${ }^{29}$. Dans ce cas, le pavillon, qui a une hauteur de 3,35 $\mathrm{m}$ (sans la chape) et une largeur d'environ $5,50 \mathrm{~m}$, devait indéniablement être prévu pour se positionner au-dessus du lit, car ses dimensions aurait été bien trop grandes pour une chaire. Il était en effet coutumier de placer un dais au-dessus des ciels des lits royaux ou princiers, comme l'atteste Philibert Delorme : "Ce qui doit être à propos du odé [haut dais] qu'on mect par dessus le lict royal, auquel on accomode parfois des rideaux de toile d'or ou d'autre matière, ainsi que Leur Majesté le requiert ${ }^{30}$.» Dais et pavillons se trouvaient - comme le ciel pourvus de pentes.

\section{Les pentes}

Dans le Thrésor de la langue françoyse de Nicot figure simplement l'indication: "les pentes d'un lict ", sans plus de précision; plus tard, dans la quatrième édition du Dictionnaire de l'Académie française, on peut lire la définition suivante: «se dit aussi 
d'Une bande qui pend autour du ciel du lit, sur le haut des rideaux. Les pentes du lit. Pentes de velours, de damas. Pentes garnies de crépines, de franges, etc. Les pentes de dehors. Les pentes de dedans, ou autrement, Les petites pentes. » Si la distinction entre petites pentes, pentes du dehors et pentes du dedans n'apparaît pas dans les sources, la définition correspond à l'usage qui est fait du mot au $\mathrm{XvI}^{\mathrm{e}}$ siècle. Il peut être alors écrit avec un « $\mathrm{h} »$ : «penthe» ou "panthe». Les pentes sont rarement décrites seules, et accompagnent le ciel dans les inventaires. La précision " pentes doublées » nous semble signifier la présence de pentes intérieures et extérieures, ce qui permettait de dissimuler les tringles et anneaux nécessaires à l'accrochage des courtines.

\section{Dossier ou dosseret}

Le dosseret est la partie qui, au niveau du chevet de tête, isole en quelque sorte le lit du mur. Souvent cité dans la continuité des différentes parties d'une parure de lit, il apparait rarement seul mais on le trouve parfois simplement décrit avec un ciel ou un dais. Il existe en effet des dosserets pour les ciels de dais.

Le terme de dosseret n'apparaît ni dans l'ouvrage de Jean Nicot, ni dans les dictionnaires de l'Académie française, postérieurs, ni dans le Dictionnaire de Trévoux. Il semblerait que ce terme soit une déformation de « dociel».

En revanche, au chapitre "dossier» du Thresor de la langue françoyse, on trouve la nuance suivante : «Dossier, C'est ce qui est fait pour appuyer le dos, Dorsuarium, Comme si vous disiez Dorsier. Selon ce on dit un banc à dossier, ou une chaire à dossier, esquels y a un appuyoir du dos : et moins proprement un dossier de pavillon pour la piece d'iceluy qui pend sur le dossier et chevet du chalit ${ }^{31}$."

En 1762, dans le Dictionnaire de l'Académie française, la définition est claire : «On appelle aussi Dossier d'un lit, Une pièce de bois fort large qui joint les deux colonnes de derrière. C'est encore la pièce d'étoffe qui couvre le derrière du lit. » Cette acception est donc valable pendant toute l'époque moderne pour les termes "dossier ", "dociel» ou « dosseret ».

\section{Recouvrir la couche}

Plusieurs termes semblent désigner le dessus de lit. La différence entre courtepointe, couverture, couverte et mante est assez ténue.

n trouve la liste d'une série de courtepointes dans l'inventaire royal de 1542, à partir du n ${ }^{\circ} 111$ : « Neuf courtepoinctes de taffetas de plusieurs couleurs, c'est assavoir une de taffetas bleu à deux endroictz picqué, à bastons rompuz, contenant une aulne deux tiers en carré $^{32}$.» Ce terme de courtepointe apparaît souvent orthographié " courtepoincte " ou "contrepointe». Selon le Dictionnaire de l'Académie française de 1694, la courtepointe est une « Couverture de parade eschancrée et piquée avec ordre et proportion ». Il s'agit donc d'un dessus de lit d'aspect plus soigné que les autres.

Les sources citent également des couvertures ou couvertes, comme dans l'inventaire de la duchesse d'Albret, où il est question de « La couverture d'une couete de satin blanc et cramoisy, à neuf bandes, chascune bande de demy satin de largeur, et de longueur contenant une aulne deux tiers $\aleph^{33}$. Le terme est probablement à prendre ici pour housse. En revanche, dans l'inventaire de Catherine de Médicis, les mentions d'« une 
couverte de taffetas blanc piquée, une couverte de toille de Hollande piquée » semblent bien désigner des dessus de lit d'aspect moins élaboré (des dessus de lit moins raffinés ?) que la courtepointe.

Enfin, les courtepointes peuvent être complétées par une "mante ». Si ce terme relève plutôt de l'habillement, il apparaît dans les sources et semble désigner un dessus de lit supplémentaire aux proportions plus petites que la courtepointe. Dans l'inventaire des ducs de Lorraine de 1543, il est fait mention pour le lit du duc Antoine et de la duchesse Renée de Bourbon d'« une contrepoincte de taffetas violet » et d'« une mante taincte en escarlatte $»^{34}$.

\section{Le tour de lit}

51 Si la mention de "tour de lit " n'est pas fréquente dans les sources de la première moitié du siècle, elle tend à croître dans la seconde moitié. Son sens a d'ailleurs évolué au fil du temps. Le mot «tour de lit » désigne d'abord les longues pièces d'étoffe assez étroites qui servent à orner le pourtour du châlit et à le dissimuler. La mention de " soubassement » a la même acception. Le tour de lit est visible sous la courtepointe et la prolonge. En revanche, il est dissimulé par les courtines lorsque celles-ci sont tirées. Le tour de lit décrit pour le meuble créé, en 1539, pour la venue de Charles Quint en France mesure $572 \mathrm{~cm}$ de long pour une hauteur de $50 \mathrm{~cm}$ : une longue pièce de velours qui permettait de couvrir tout le pourtour du lit ${ }^{35}$. La plupart des tours de lit mesurent entre 50 et $55 \mathrm{~cm}$ de haut, ce qui correspond à un lé d'étoffe.

Pourtant le mot « tour de lit » semble embrasser une autre acception dans la deuxième moitié $d u \mathrm{XvI}^{\mathrm{e}}$ siècle. Par exemple, dans l'inventaire de Catherine de Médicis, est décrit " ung tour de lict de serge de Beauvais blanche, contenant cinq lez et une bande sur deux aulnes moings demy tiers de hault, le bas frangé d'une soye blanche " puis « ung autre tour de lit de serge blanche de quatre lez et demy de large sur une aulne deux tiers de hault, ung dossier de mesme d'un lez et demy de mesme hauteur $\aleph^{36}$. Suivent d'autres tours de lit aux dimensions approchantes. Compte tenu des dimensions relativement hautes, ici «tour de lit» doit être compris comme synonyme de « housse » qui entoure l'ensemble du lit de manière à le protéger. Le tissu employé, un sergé blanc, confirme cette hypothèse.

\section{Les courtines ou rideaux}

$53 \mathrm{Au} \mathrm{XVI}{ }^{\mathrm{e}}$ siècle, les termes de courtine et de rideau sont utilisés indifféremment pour désigner le même élément. En 1694, le Dictionnaire de l'Académie française donne pour courtine la définition suivante: "Rideau de lit. Courtine de damas. fermer, tirer les courtines", insistant ainsi sur la synonymie des deux $\operatorname{mots}^{37}$. La définition se poursuit par : « En ce sens il est vieux », ce qui atteste de l'usage ancien du mot courtine.

Dans l'inventaire de la duchesse d'Albret, certains rideaux sont mentionnés séparément d'autres éléments de lits, et dans ce cas leur usage est précisé comme ces « deux grans rideaulx de lict de taffetas vert $»^{38}$.

Dans l'inventaire de Catherine de Médicis, les rideaux sont nombreux et l'on note la présence du terme de "bonne grâce " à plusieurs reprises. Cette expression semble apparaître dans les deux dernières décennies du XVI ${ }^{e}$ siècle, sans qu'il soit possible de dire si elle désigne des pièces réellement distinctes des rideaux. Parfois, il semble 
évident que bonnes grâces et rideaux soient bien distincts, comme au n ${ }^{\circ} 450$ : « ung ciel à doubles pentes de réseuil remply de soye de plusieurs couleurs, meslé d'or et d'argent, garny de six pentes, trois soubassemens, trois grans rideaux, deux bonnes graces, ung fond et ung dociel, les pentes, fond et dociel doublez de satin blanc, et la couverte de parade doublée de mesme ${ }^{39}$.» Or, ce terme de "bonne grâce» est absent des dictionnaires avant la quatrième édition de celui de l'Académie française qui le définit comme «lez d'étoffe qu'on attache vers le chevet et vers les pieds d'un lit, pour accompagner les grands rideaux $»^{40}$. Les documents attestent pourtant qu'il était en usage dès la fin du Xvi ${ }^{e}$ siècle.

Enfin, le terme de garniture peut être ponctuellement employé pour désigner l'intégralité des parties textiles. Par exemple, dans l'inventaire royal de 1542, il est question de la "garniture d'un lict de velours cramoisy, enrichy de brancaille de toille d'or avec feuillaige de mesmes et fueillaige de lyere; les graynes dudict lyere faictes de barroques de perles ", qui inclut un dosseret, le ciel et cinq pentes ${ }^{41}$.

C'est cette signification qu'il faut aussi comprendre par l'usage du verbe "garnir » dans l'inventaire de Charlotte d'Albret où il est question d'" ung lict garny de coette, coessin, une mante une couverture de drap rouge et jaulne, le ciel de tappicerie à franges jaulnes, rouges et vertes, et les courtines de serge de pareille couleur $»^{42}$.

Si le vocabulaire descriptif des éléments menuisés est encore relativement sommaire, les différents termes désignant les parties textiles des lits d'apparat au $\mathrm{XvI}^{\mathrm{e}}$ siècle reflètent une réalité : celle de la place prépondérante des étoffes, tant dans l'économie de l'ameublement que de l'apparat de la demeure. Ce vocabulaire progressivement fixé à la Renaissance deviendra la norme pour décrire les lits au XvII ${ }^{\mathrm{e}}$ siècle.

\section{NOTES}

1. - CORROZET, Gilles. Les Blasons domestiques contenantz la décoration d'une maison honneste, et du mesnage estant en icelle,... Paris : G. Corrozet, 1539, fol. 16v-17v. Consultable sur Gallica.

2. - Cette question avait été l'objet de la réunion du Comité lit du 12 mars 2015 au C2RMF (Paris). Nous remercions Caroline Vrand des éléments précieux qu'elle a apportés à la discussion.

3. - Paris, BnF. Français 22335, Inventaires des meubles et des archives de diverses princesses et princes français. ( $\mathrm{XV}{ }^{e}$-XVII ${ }^{e}$ siècle). Inventaires des tapisseries, tableaux, reliquaires, meubles et « acoustremens » de la reine Anne de Bretagne, 1495-1511 (p. 1 et suiv.).

4. - AN, MC/CXXII/1282; voir SCHNEEBALG-PERELMAN, Sophie. «Richesse du garde-meuble parisien de François $\mathrm{I}^{\text {er }}$. Inventaires inédits de 1542 et 1551 ». Gazette des beaux-arts, 1971, II, p. 253-304, nos $335-342$.

5. - NICOT, Jean. Thresor de la langue françoyse tant ancienne que moderne... Paris : David Douceur, 1606, p. 374, voir le site: http://portail.atilf.fr/cgi-bin/dico1look.pl? strippedhw=lict\&submit=\&dicoid=NICOT1606 [consulté le 27/06/2019].

6. - AN, MC/CXXII/1282; voir SCHNEEBALG-PERELMAN, Sophie. «Richesse du garde-meuble parisien de François $\mathrm{I}^{\mathrm{er}}$... ». Art. cit. 
7. - HAVARD, Henri. Dictionnaire de l'ameublement et de la décoration: depuis le XIII siècle jusqu'à nos jours. Paris : Maison Quantin, [1886-1887], vol. 1, col. 682 ; sur la polysémie du mot « chambre » et sur l'histoire de la pièce, voir PERROT, Michelle. Histoire de chambres. Paris : Seuil, 2009.

8. - NICOT, Jean. Thresor de la langue françoyse tant ancienne que moderne... Op. cit., p. 116, voir le site : http://portail.atilf.fr/cgi-bin/dico1look.pl?strippedhw=chaslit\&submit=\&dicoid=NICOT1606 [consulté le 27/06/2019].

9. - AN, MC/CXXII/1282 ; voir SCHNEEBALG-PERELMAN, Sophie. «Richesse du garde-meuble parisien de François $\mathrm{I}^{\mathrm{er}}$... ». Art. cit., $\mathrm{n}^{\circ} 142$.

10. - NICOT, Jean. Thresor de la langue françoyse tant ancienne que moderne... Op. cit., p. 153, voir le site :

http://portail.atilf.fr/cgi-bin/dico1look.pl?

strippedhw=couche\&headword=\&docyear=ALL\&dicoid=ALL\&articletype=1 [consulté le 27/06/2019].

11. - GRODECKI, Catherine. Histoire de l'art au XVI siècle (1540-1600). Paris : Archives nationales, 1985-1986, t. 1, Architecture, vitrerie, menuiserie, jardins, p. 263.

12. - GODEFROY, Théodore. Le Cérémonial françois... Paris : Sébastien Cramoisy, 1649, vol. 2, p. 153.

13. - SCHNEEBALG-PERELMAN, Sophie. "Richesse du garde-meuble parisien de François I ${ }^{\mathrm{er}} . .$. ». Art. cit., $\mathrm{n}^{\circ} 335$ sqq. Nous remercions ici Monique Chatenet pour les fructueux échanges sur les dimensions des lits que nous avons eus.

14. - Commande de mobilier royal par Jacques Patin, peintre du roi, à Noël Biard, maître menuisier à Paris, 19 juillet 1584, Paris, AN, MC/LIV/110.

15. - SCHNEEBALG-PERELMAN, Sophie. "Richesse du garde-meuble parisien de François I ${ }^{\mathrm{er}} . .$. ». Art. cit., $\mathrm{n}^{\circ} 142$.

16. - Ibid., $\mathrm{n}^{\circ} 330$.

17. - BONNAFFÉ, Edmond. Inventaire de la duchesse de Valentinois Charlotte d'Albret-1514. Paris : A. Quantin, 1878, nos 375 et 379.

18. - Marché par lequel Pierre Langevin, compagnon menuisier, s'engage à faire pour Jean Malingre, avocat au Parlement, $1^{\mathrm{er}}$ août 1547, Paris, AN., MC/XIX/172.

19. - En particulier, dans les comptes de l'argenterie de Charles VIII (1488-1492). Paris, AN, KK 70, KK 71 et KK 72.

20. - HUGUET, Edmond. Dictionnaire de la langue française du XVI siècle. Paris : Édouard Champion, 1925-1967.

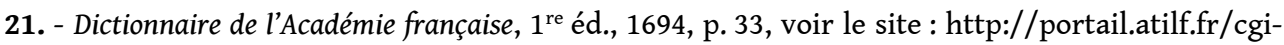
bin/dico1look.pl?strippedhw=couche\&headword=\&docyear=ALL\&dicoid=ALL\&articletype=1 [consulté le 27/06/2019].

22. - AN, MC/CXXII/1282 ; SCHNEEBALG-PERELMAN, Sophie. « Richesse du garde-meuble parisien de François $I^{\text {er }}$... ». Art. cit., $\mathrm{n}^{\text {os }} 324-328$ puis $\mathrm{n}^{\circ} \mathrm{LXXXVI.}$

23. - Dictionnaire de l'Académie française, $1^{\text {re }}$ éd., 1694, p. 279, voir le site : http://portail.atilf.fr/cgibin/dico1look.pl?strippedhw=paillasse\&headword=\&docyear=ALL\&dicoid=ALL\&articletype=1 [consulté le 27/06/2019].

24. - AN, MC/CXXII/1282 ; SCHNEEBALG-PERELMAN, Sophie. «Richesse du garde-meuble parisien de François $\mathrm{I}^{\mathrm{er}} . .$. ». Art. cit., $\mathrm{n}^{\mathrm{os}} \mathrm{n}^{\mathrm{os}} 10-13$; voir aussi Meubles de la reine mère Louise de Savoie, 1518-1521, Paris, AN, KK 90 ; Une Reine sans couronne. Louise de Savoie, mère de François I ${ }^{\text {er }}$. Cat. exp., musée national de la Renaissance, Écouen, 13 octobre 2015-26 janvier 2016. Paris : RMN-GP, 2015, cat. 40 ; FAGNART, Laure. "Louise de Savoie et la Chambre des Bucoliques». Dans BRIOIST, Pascal, FAGNART, Laure, MICHON, Cédric (dir.). Louise de Savoie 1476-1531. Tours/Rennes : Presses universitaires François Rabelais/PUR, 2015, p. 205-218.

25. - BONNAFFÉ, Edmond. Inventaire des meubles de Catherine de Médicis 1589. Paris : Auguste Aubry, 1874, no 10.

26. - Comptes de l'argenterie de Charles VIII (1488-1492), Paris, AN, KK 71. 
27. - AN, MC/CXXII/1282 ; SCHNEEBALG-PERELMAN, Sophie. « Richesse du garde-meuble parisien de François $\mathrm{I}^{\mathrm{er}}$... ». Art. cit., $\mathrm{n}^{\text {os }}$ 84-87.

28. - Dictionnaire de l'Académie française, $1^{\text {re }}$ éd., 1694, p. 190, voir le site : http://portail.atilf.fr/cgibin/dico1look.pl?strippedhw=ciel\&headword=\&docyear=ALL\&dicoid=ALL\&articletype=1 [consulté le 28/06/2019].

29. - Ibid. $\mathrm{n}^{\text {os }} 335-342$; voir BARBIER, Muriel. «Quelles étoffes pour les lits des grandes occasions dans la France de la Renaissance? ». Dans COUPRY, Claude, COUSIN, Françoise (dir.). Étoffes des grands jours. Actes des journées d'étude 2017, Fontainebleau. Paris : AFET, 2018, p. 40.

30. - DELORME, Philibert. Le Premier tome de l'Architecture. Paris : Fédéric Morel, 1568, livre IX, chap. I.

31. - NICOT, Jean. Thresor de la langue françoyse tant ancienne que moderne... Op. cit., p. 212, voir le site : $\quad$ http://portail.atilf.fr/cgi-bin/dico1look.pl? strippedhw=dossier\&headword=\&docyear=ALL\&dicoid=ALL\&articletype=1 [consulté le 28/06/2019].

32. - AN, MC/CXXII/1282 ; SCHNEEBALG-PERELMAN, Sophie. « Richesse du garde-meuble parisien de François I ${ }^{\mathrm{er}}$... ». Art. cit., $\mathrm{n}^{\circ} 111$ sqq.

33. - BONNAFFÉ, Edmond. Inventaire de la duchesse de Valentinois...Op. cit., $\mathrm{n}^{\circ} 331$.

34. - Voir dans ce numéro : BARBIER, Muriel et PÉNET, Pierre-Hippolyte. « Le lit du duc Antoine de Lorraine et de la duchesse Renée de Bourbon : une nouvelle vie grâce au numérique ", In Situ [En ligne], 40|2019, mis en ligne le 15 septembre 2019, consulté le 30 septembre 2019. URL: http://journals.openedition.org/insitu/24089.

35. - SCHNEEBALG-PERELMAN, Sophie. «Richesse du garde-meuble parisien de François I ${ }^{\mathrm{er}} . .$. ». Art. cit., $\mathrm{n}^{\circ} 335$ sqq.

36. - BONNAFFÉ, Edmond. Inventaire des meubles de Catherine de Médicis. Op. cit., $\mathrm{n}^{\text {os }} 118$ et 119.

37. - Dictionnaire de l'Académie française, $1^{\text {re }}$ éd., 1694, p. 274, voir le site : http://portail.atilf.fr/cgibin/dico1look.pl?strippedhw=courtine\&headword=\&docyear=ALL\&dicoid=ALL\&articletype=1 [consulté le 28/06/2019].

38. - BONNAFFÉ, Edmond. Inventaire de la duchesse de Valentinois... Op. cit., $\mathrm{n}^{\circ} 383$.

39. - BONNAFFÉ, Edmond. Inventaire des meubles de Catherine de Médicis. Op. cit., $\mathrm{n}^{\circ} 450$.

40. - Dictionnaire de l'Académie française, $4^{\mathrm{e}}$ éd., 1762, t. I, p. 160, voir le site : http://portail.atilf.fr/ cgi-bin/dico1look.pl?strippedhw=bonne+gr\%E2ce [consulté le 28/06/2019].

41. - AN, MC/CXXII/1282 ; SCHNEEBALG-PERELMAN, Sophie. « Richesse du garde-meuble parisien de François $\mathrm{I}^{\mathrm{er}} \ldots$... Art. cit. $\mathrm{n}^{\mathrm{os}} 296-298$.

42. - BONNAFFÉ, Edmond. Inventaire de la duchesse de Valentinois... Op. cit., $\mathrm{n}^{\circ} 455$.

\section{RÉSUMÉS}

Si quelques éléments de lits de la Renaissance sont conservés, force est de constater qu'aucun lit complet de la période allant du milieu du Xve siècle à l'aube du XVII ${ }^{\mathrm{e}}$ siècle ne nous est parvenu. Les sources (inventaires après décès, récits officiels, correspondances et contrats) sont donc le seul moyen de connaître l'aspect des lits. Or les termes employés dans ces documents sont bien souvent éloignés de ceux du français d'aujourd'hui. À partir de documents d'archives reflétant essentiellement les intérieurs royaux ou princiers, pour la plupart déjà publiés, il est ici proposé un décryptage du vocabulaire relatif aux lits de la Renaissance. Au-delà de la polysémie du mot 
« lit », les divers termes servant à désigner le bâti, les parties textiles mais aussi les éléments composant le coucher sont étudiés.

A few parts of some Renaissance beds have been preserved in France but not a single complete bed from the period starting in the middle of the fifteenth century and finishing at the beginning of the seventeenth has survived. Archival sources such as inventories after death, official reports, correspondence and contracts are consequently the only way of gaining some idea of what these beds looked like. But the terms used in these documents are far removed from present-day French. Based on archive documents (most of them already published) which primarily concern royal or princely interiors, this article proposes a decoding of the vocabulary pertaining to Renaissance beds. Beyond the polysemy of the word 'bed' itself, we look at the diversity of terms used to designate the bed frame, the textile elements and other elements constituting this piece of furniture for sleeping in.

INDEX

Keywords : bed, sixteenth century, vocabulary, furniture, inventory after death, habitat, sixteenth-century textile

Mots-clés : lit, xvie siècle, vocabulaire, ameublement, inventaire après décès, habitat, textile xvie siècle

\section{AUTEUR}

MURIEL BARBIER

Conservateur du patrimoine, Mobilier national muriel.barbier@culture.gouv.fr 